\title{
Experimental assessment of the representative strains in instrumented sharp indentation
}

\author{
N. Chollacoop ${ }^{a}$, U. Ramamurty ${ }^{b, *}$ \\ ${ }^{a}$ National Metal and Materials Technology Center (MTEC), 114 Paholyothin Road, Pathumthani 12120, Thailand \\ ${ }^{\mathrm{b}}$ Department of Metallurgy, Indian Institute of Science, Bangalore 560 012, India
}

\begin{abstract}
Experimental assessment of the reverse algorithms that enable the extraction of plastic properties from the load-depth of penetration curves was conducted. Results show that they predict the stresses at 3.3\% and 5.7\% representative strains for Berkovich and $60^{\circ}$ cone-equivalent three-sided pyramidal indenters, respectively, with good accuracy. It was shown that the uniaxial stress-strain curves could be reconstructed from the indentation data.
\end{abstract}

Keywords: Microindentation; Plastic deformation; Tension test; Hardness; Representative strain

\section{Introduction}

Instrumented indentation is a widely-used technique to evaluate the elastic modulus, $E$, and the hardness, $H$, from the load, $P$, vs. depth of penetration, $h$, data. However, extraction of the yield strength, $\sigma_{y}$ and the work hardening exponent, $n$, from the $P-h$ curves of sharp indenters is not done as routinely, due to the lack of reliable and simple-to-implement methodologies. Recently, Dao et al. [1] and Chollacoop et al. [2] have conducted computational studies within the framework of large-strain finite element analysis (LFEA) for sharp indentation on power-law hardening elastic-plastic solids and identified a set of analytical functions that take the pile-up/sink-in effects into account. Further, they identified a representative plastic strain, $\varepsilon_{\mathrm{r}}$ for each indenter geometry as a strain level that allows for the description of the indentation loading response independent of $n$. Estimated values of $\varepsilon_{\mathrm{r}}$ are $3.3 \%$ and $5.7 \%$ for

\footnotetext{
* Corresponding author. Tel.: +91 802 2933241; fax: +91 802 3600472.

E-mail address: ramu@met.iisc.ernet.in (U. Ramamurty).
}

Berkovich and $60^{\circ}$ cone-equivalent pyramidal indenters, respectively. On this basis, they developed algorithms to assess properties of materials within the context of single and dual indentations. These algorithms promise an opportunity to extract $\sigma_{y}$ and $n$ from the $P-h$ curves with similar ease to $E$ and $H$ measurement. However, a critical experimental assessment of them is necessary before they can be fully deployed. This is particularly important since the reported values of $\varepsilon_{\mathrm{r}}$ in the literature are significantly different. Tabor [3] proposed that it is $\sim 8 \%$ within the plastically deformed region of a Vickers indent. Johnson's expanding cavity model shows that $\varepsilon_{\mathrm{r}}=0.2 \cot \left(70.3^{\circ}\right)$ for Vickers indenter [4]. Small-strain finite element simulations of Giannakopoulos et al. [5] suggest that the $\varepsilon_{\mathrm{r}}$ for Vickers and Berkovich indenters is $\sim 29 \%$, which is in agreement with the maximum subsurface strain observed in the region adjacent to the tip of Vickers indentation of $\mathrm{Cu}$ by Chaudhri [6]. Atkins and Tabor [7] estimated $\varepsilon_{\mathrm{r}}$ values as a function of the cone angle through hardness measurements on copper and mild-steel samples that are work hardened to different levels prior to indentation. These are plotted, along with those estimated by Chollacoop et al. [2], as a 


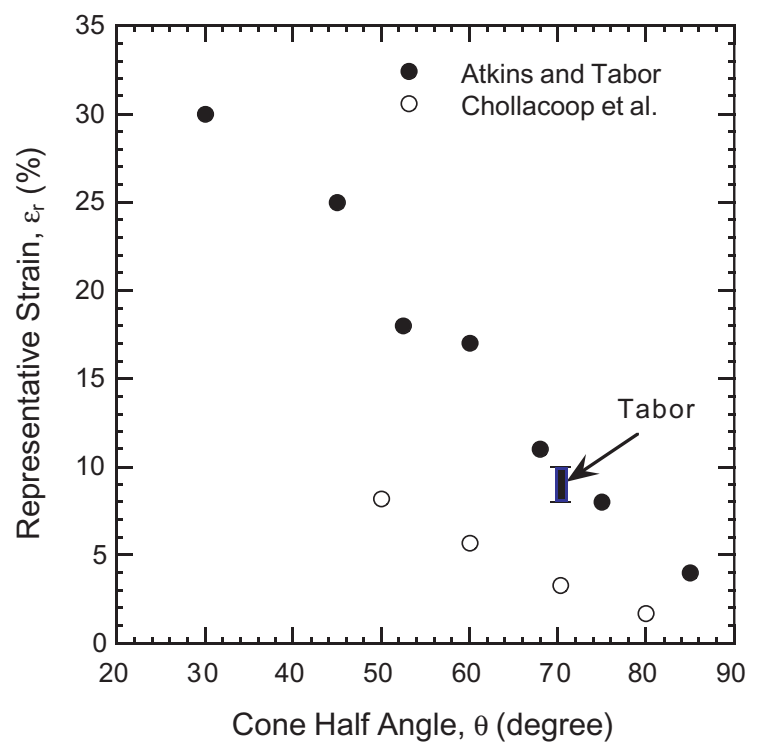

Fig. 1. Variation of the representative strain, $\varepsilon_{\mathrm{r}}$, with the indenter tip half angle, $\theta$.

function of the cone angle in Fig. 1. As seen, the $\varepsilon_{\mathrm{r}}$ values reported in these two papers are significantly different. Since the concept of $\varepsilon_{\mathrm{r}}$ is critical to the accuracy of the $\sigma_{y}$ and $n$ estimates, a close experimental examination is necessary.

\section{Experimental}

The following experimental procedure, illustrated in Fig. 2, is adopted. Let us suppose that a given ductile metal is indented with Berkovich or a Vickers indenter and the $P-h$ data are analyzed using the methodology of Dao et al. [1] to estimate the stress at 3.3\% plastic strain, $\sigma_{0.033}$. Let the as-received material be subjected

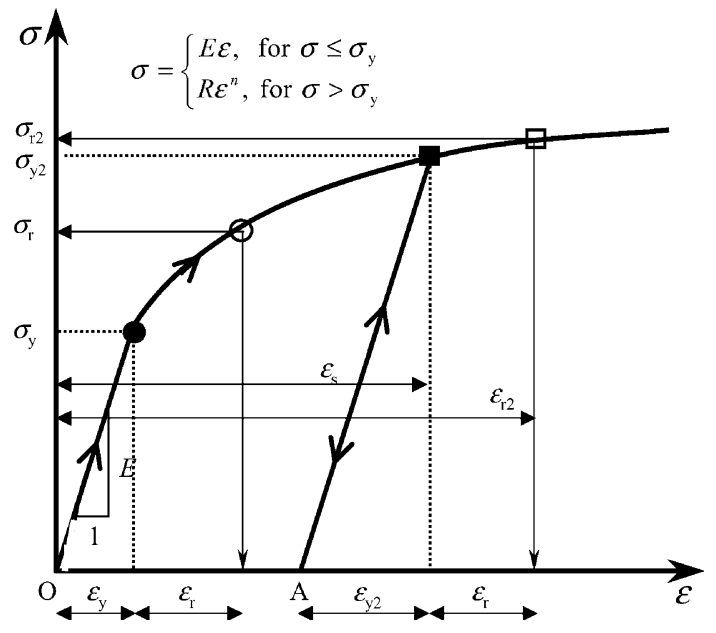

Fig. 2. Schematic illustration of the experimental procedure utilized to assess the reverse algorithms. to a prior strain of $\varepsilon_{\mathrm{s}}$ (with $\varepsilon_{\mathrm{s}}>$ the yield strain, $\varepsilon_{y}$ ). Analysis of the $P-h$ curves generated from the prestrained material should yield a stress $\sigma_{\mathrm{r} 2}$ that corresponds to a strain of $\varepsilon_{\mathrm{r} 2}$ where $\varepsilon_{\mathrm{r} 2}=\varepsilon_{\mathrm{r}}+\varepsilon_{\mathrm{s}}$ since logarithmic plastic strains are additive. In other words, the prior plastic strain simply translates the origin of the $\sigma-\varepsilon$ plot from point $\mathrm{O}$ to point $\mathrm{A}$ by an amount equal to $\varepsilon_{\mathrm{s}}-\varepsilon_{y 2}$, as shown in Fig. 2. Thus, by indenting specimens that were subjected to different levels of $\varepsilon_{\mathrm{S}}$ and subsequently analyzing the data, it should be possible to construct the $\sigma-\varepsilon$ curve, which can then be compared to that obtained from a tensile test to assess the accuracy of the predictions. Note that this procedure is similar to that employed by Atkins and Tabor [7] although the maximum strain scale they have used $(\sim 138 \%)$ is an order of magnitude larger than that used $(\sim 10 \%)$ in the present study.

Two materials were used for the experimental investigation: a highly cold-worked pure copper and an $\mathrm{Al}$ 6061-T651 alloy, both in the form of $4.8 \mathrm{~mm}$ thick plates. The former exhibits elastic-perfectly plastic behavior whereas the latter strain hardens considerably. Therefore, the Al alloy should exhibit steeper $P-h$ curves with increasing $\varepsilon_{\mathrm{s}}$ whereas they should be independent of $\varepsilon_{\mathrm{s}}$ in the cold-worked $\mathrm{Cu}$.

Four dog-bone specimens with $\sim 50 \mathrm{~mm}$ gage length and $\sim 9.5 \mathrm{~mm}$ gage width were machined from each plate. The Al specimens were fully annealed in atmosphere at $415^{\circ} \mathrm{C}$ for $2.5 \mathrm{~h}$ (O temper) to obtain the highest hardening. Uniaxial tensile tests were conducted in a universal testing machine at a crosshead speed of $1.27 \mathrm{~mm} / \mathrm{min}$ while the strains were monitored with a $25 \mathrm{~mm}$ gage length extensometer. The $\mathrm{Cu}$ specimens were strained to $\sim 1.6 \%, 4 \%, 7.3 \%$ and $10 \%$ engineering strains each before unloading (labeled $\mathrm{Cu} 1, \mathrm{Cu} 2, \mathrm{Cu} 3$ and $\mathrm{Cu} 4$, respectively) whereas the $\mathrm{Al}$ specimens were strained to $\sim 0.8 \%, 3 \%, 5 \%$ and $7 \%$ engineering strains each before unloading (labeled A11, A12, A13 and A14, respectively).

One centimeter length indentation specimens were machined from the gage section of each strained specimen and from the grips of $\mathrm{Cul}$ and All (labeled Culgs and Allgs, respectively). All the specimens were polished to a $1 \mu \mathrm{m}$ finish with diamond paste. The Culgs and Allgs were polished deeper than other specimens to completely remove the surface layer that is strained due to gripping and were utilized to evaluate the as-received material's indentation response.

The polished samples were then indented using an instrumented microindenter (MicroMaterials, Wrexham, UK) with Berkovich and $60^{\circ}$ cone equivalent three-sided pyramid diamond tips at a loading/unloading rate of $0.1 \mathrm{~N} / \mathrm{s}$. A $10 \mathrm{~s}$ hold period at the maximum load is utilized to assess the thermal drift and subsequently subtract it from the measured $P-h$ curves. The tip geometries were calibrated through the standard area 
function calibration. The spacing between the consecutive indents was maintained to be at least 10 times the approximate indentation radius. Limited by the maximum depth and load ratings of the instrument, the $\mathrm{Cu}$ and $\mathrm{Al}$ specimens were indented with the maximum load of 12 and $4 \mathrm{~N}$, respectively under the Berkovich tip. In the case of the $60^{\circ}$ cone equivalent three-sided pyramid tip, they were indented to maximum loads of 4 and $2 \mathrm{~N}$, respectively. In all cases, at least six indentation tests were conducted for each case to ensure repeatability.

It is important to note here that the $h$ recorded by the instrumented indenter also includes the displacement associated with the load train as a result of the machine compliance. The machine compliance $(0.34 \mu \mathrm{m} / \mathrm{N})$ was determined by indenting fused silica with known $E$, and the $h$ associated with this was subtracted from the experimental data and the modified $P-h$ curves were analyzed using the reverse algorithms.

\section{Results}

The experimental tensile load-displacement plots were converted to true stress, $\sigma$, and true strain, $\varepsilon$, data and plotted in Fig. 3, which shows that the $\mathrm{Cu}$ samples exhibit an elastic-perfectly plastic behavior with a nearzero value of $n$ whereas the annealed Al sample workhardens with $n$ of $\sim 0.3$. The average $E, \sigma_{y}$ and $n$ values measured from these tests are listed in Table 1.

Fig. 4(a) and (b) shows representative $P-h$ curves obtained from the $\mathrm{Cu}$ and $\mathrm{Al}$ specimens, respectively. Dao et al. [1] and Chollacoop et al. [2] have rigorously shown that the loading curvature of the indentation response depends only on the $E$ and the representative stress. Thus, the low strain hardening observed in $\mathrm{Cu}$ samples

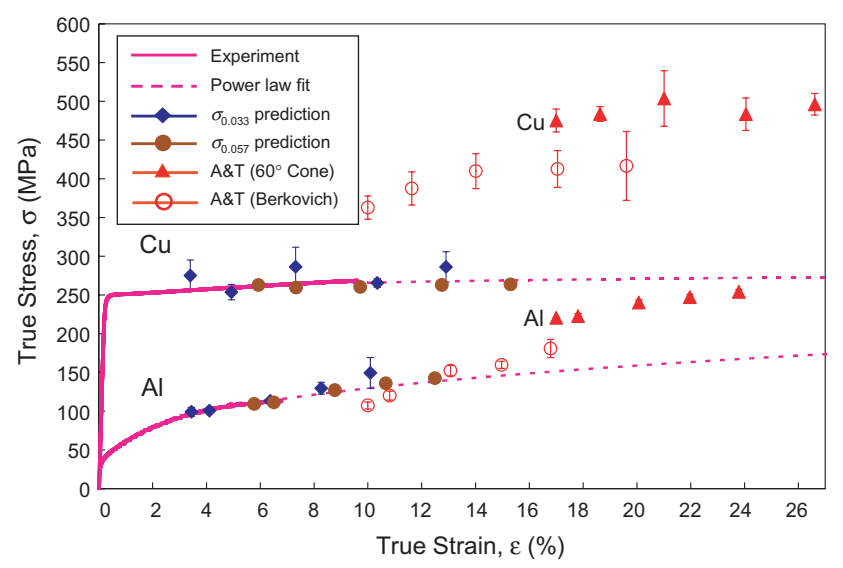

Fig. 3. True stress-true strain $(\sigma-\varepsilon)$ curves generated through the uniaxial tensile tests. The values of the representative stresses, extracted using the reverse algorithms or by using the Atkins and Tabor (referred to A\&T in the legends) method and offset by the prior plastic strain, are also plotted for comparison. implies equal values of representative stress and hence similar indentation curves. Conversely, strain hardening in the $\mathrm{Al}$ samples should lead to higher curvature for the loading part of the $P-h$ curves with increasing $\varepsilon_{\mathrm{s}}$. The experimental $P-h$ curves are consistent with these expectations.

The individual $P-h$ data were analyzed using the single and dual indenter reverse algorithms detailed in Refs. [1,2], respectively. The $E$ values, extracted from the Berkovich indentation data and listed in Table 1, match closely with those measured in the tensile tests. Extracted values of $\sigma_{0.033}$ and $\sigma_{0.057}$ were plotted in Fig. 3 offset by the $\varepsilon_{\mathrm{s}}$ to which the specimen was subjected prior to indentation. It is seen that the $\sigma-\varepsilon$ curves are well captured by the predictions from the reverse algorithms. In particular, the $\sigma_{0.057}$ predictions allow for the construction of the $\sigma-\varepsilon$ curves remarkably well, indicating the robustness of this prediction. Relatively large scatter seen in $\sigma_{0.033}$ is possibly due to the relatively blunt Berkovich tip (radius $\sim 250 \mathrm{~nm}$ ), a result of being used extensively prior to this study. On the other hand, the $60^{\circ}$ cone equivalent three-sided pyramid tip was custom-made for this particular study and hence sharp (radius $\sim 50 \mathrm{~nm}$ ). Since indentation using a blunted tip is like indenting into a harder material, it is suspected that the tip-blunting is the reason for the larger scatter in the Berkovich data. Although the tip radius effect will be significant only when the indentation depth (or contact radius) is comparable to the radius of tip curvature, it is likely to alter the Kick's exponent (which is implicitly assumed to be equal to two in the reverse algorithms when fitting the loading portion of the $P-h$ curves). Further, it also affects the point of initial contact, shifting the entire loading portion and not just at $h<$ tip radius, leading to errors in the estimation of the loading curvature, $C$. Note that Dao et al.'s [1] analysis (Table 7 of their paper) shows that a small error of $\pm 2 \%$ in $C$ could lead to about $\pm 10 \%$ error in $\sigma_{0.033}$ estimate. This could be another reason for a large scatter in the Berkovich data.

A least-squares fit of the power law through the $\sigma_{0.033}$ and $\sigma_{0.057}$ data was used to estimate the values of $\sigma_{y}$ and $n$, which are listed in Table 1. Good agreement between the predicted and experimental values, for both the $\mathrm{Cu}$ and the Al samples, is noted. Although the discrepancy between the predicted and experimental values of $\sigma_{y}$ for $\mathrm{Al}$ and $n$ for $\mathrm{Cu}$ appear large in terms of difference in $\%$, the agreement can be considered good given the low absolute values in these cases.

Finally, it is instructive to compare the accuracy of the $\varepsilon_{\mathrm{r}}$ values estimated by Atkins and Tabor [7]. For this purpose, the $H$ values were estimated for both the tip geometries by dividing the maximum applied force with the indenter imprint area. Then, using the constraint factor, $S$, values given by Atkins and Tabor for these particular geometries, the flow stress is estimated and 
Table 1

Averaged mechanical properties determined from tensile tests with comparison with the extracted value from indentation analysis

\begin{tabular}{|c|c|c|c|c|c|c|c|}
\hline \multirow[t]{2}{*}{ Material } & \multicolumn{3}{|c|}{ Tensile test data } & \multirow[t]{2}{*}{$v^{\mathrm{a}}$} & \multicolumn{3}{|c|}{ Data extracted from $P-h$ curves } \\
\hline & $E(\mathrm{GPa})$ & $\sigma_{y}(\mathrm{MPa})$ & $n$ & & $E(\mathrm{GPa})$ & $\sigma_{y}(\mathrm{MPa})$ & $n$ \\
\hline $\mathrm{Cu}$ & 113 & 238 & 0.03 & 0.30 & 112 & 247 & 0.02 \\
\hline $\mathrm{Al}$ & 66 & 25 & 0.3 & 0.33 & 64.5 & 34 & 0.25 \\
\hline
\end{tabular}

${ }^{\text {a }}$ Literature value [9].
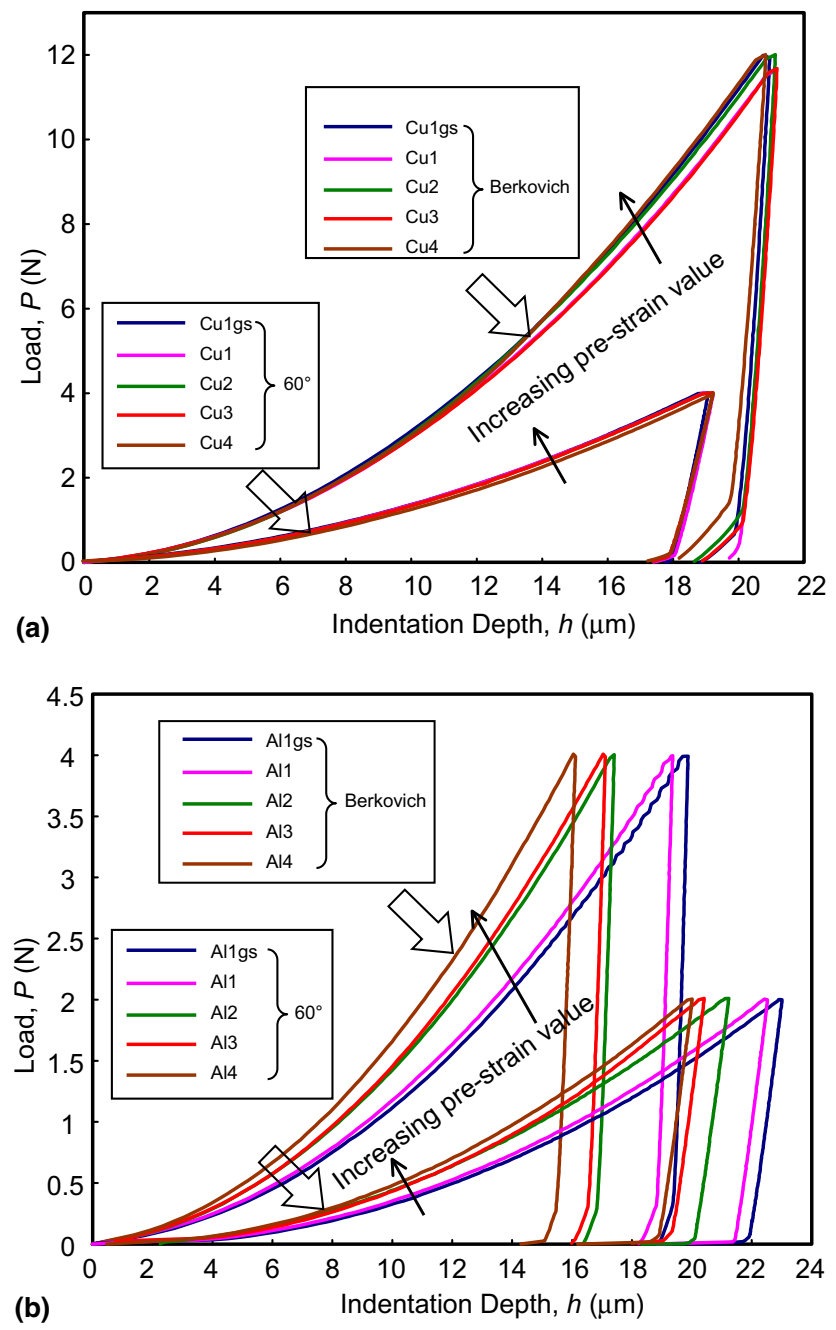

Fig. 4. Experimental indentation responses under both Berkovich and $60^{\circ}$ cone equivalent three-sided pyramid tips for (a) $\mathrm{Cu}$ and (b) $\mathrm{Al}$ specimens.

plotted in Fig. 3 offset by the $\left(\varepsilon_{\mathrm{r}}+\varepsilon_{\mathrm{s}}\right)$ with the $\varepsilon_{\mathrm{r}}$ of $10 \%$ and $17 \%$ for Berkovich and $60^{\circ}$ cone indenters. Note that this procedure is exactly the same as that used by them. As seen from Fig. 3, the estimated values are significantly larger than the corresponding extrapolated flow stresses (made assuming power-law), except for the case of Berkovich data of Al samples. Clearly, the constraint factor and hence the $\varepsilon_{\mathrm{r}}$ values estimated by Atkins and Tabor are less accurate than those made using the finite element simulations. This is probably due to the implicit assumption by Atkins and Tabor that the constraint factor $S$ for a given cone angle is independent of prior plastic strain. Subsequent work by Johnson [8] has shown that the constraint factor $S$ is highly sensitive to the $E / \sigma_{y}$ as well as the $E / H$ ratios, indicating that the basic assumption that $S$ is independent of prior plastic strain (and hence the flow stress in a work-hardening metal) made by Atkins and Tabor in their analysis is inaccurate.

Although the objective of this paper is only to assess the accuracy of the $\varepsilon_{\mathrm{r}}$ values estimated by Dao et al. [1] and Chollacoop et al. [2] on one side and by Atkins and Tabor [7] on the other, it should be noted that Dao et al. [1] demonstrate that the $\varepsilon_{\mathrm{r}}$ values of Berkovich indenter can be related to each other. They suggest that the apparent disparity is not due to discrepancies in data, constitutive modeling or fitting procedure, but from the choice of functional parameters that were used to describe the indentation process (the $P-h$ curves or the hardness). Because of the different definitions, these two methods lead to the identification of different $\varepsilon_{\mathrm{r}}$ values and the experimental exercise conducted in the present paper simply demonstrates that the $\varepsilon_{\mathrm{r}}$ values estimated by recourse to LEFA and dimensionless analysis are relatively more accurate.

\section{Summary}

Experiments were conducted to critically assess the representative strains underneath sharp indenters, estimated by Dao et al. [1] and Chollacoop et al. [2] through LFEA for Berkovich and conical indenters with varying cone angles, respectively. Two materials of contrasting plastic behavior (very low strain-hardening pure copper and very high strain-hardening annealed $\mathrm{Al}$ alloy) were plastically strained prior to indentations under both Berkovich and $60^{\circ}$ cone equivalent three-sided pyramid tips. A series of $\sigma_{0.033}$ were predicted from single indenter reverse algorithms, showing a good agreement with uniaxial tensile stress-strain curve. Better agreement was achieved for a series of $\sigma_{0.057}$ for dual indenter algorithms, possibly due to a sharper tip. This representative strain concept allows for the possibility of constructing the entire stress-strain curves, with better accuracy and less sensitivity, from multiple indentations of one or more indenter tips. 


\section{Acknowledgments}

We are grateful to Prof. S. Suresh for his support and encouragement and to Dr. Ming Dao for many useful discussions on this topic.

\section{References}

[1] Dao M, Chollacoop N, Van Vliet KJ, Venkatesh TA, Suresh S. Acta Mater 2001;49:3899.
[2] Chollacoop N, Dao M, Suresh S. Acta Mater 2003;51: 3713.

[3] Tabor D. Hardness of metals. 1st ed. Oxford: Clarendon Press; 1951.

[4] Johnson KL. J Mech Phys Solids 1970;118:115.

[5] Giannakopoulos AE, Larsson PL, Vestergaard R. Int J Solids Struct 1994:31:2679.

[6] Chaudhri MM. Acta Mater 1998;46:3047.

[7] Atkins AG, Tabor D. J Mech Phys Solids 1965;13:149.

[8] Johnson KL. Contact mechanics. Oxford: Oxford University Press; 1985.

[9] http://www.matweb.com/. 